EDITORIAL

\title{
LA CIRUGÍA BARIÁTRICA COMO TRATAMIENTO DE LA DIABETES. CONSIDERACIONES DEL EMBARAZO POSTERIOR A LA CIRUGÍA BARIÁTRICA
}

La obesidad es el desorden metabólico más frecuente considerada por la Organización Mundial de la Salud (OMS) como uno de los problemas más graves de salud mundial del siglo XXI'; la epidemia de diabetes tipo 2 (DM2) acompaña a la obesidad en lo que se denomina "epidemias mellizas".

En el año 1991, la Conferencia del NIH (National Institute of Health) reconoció a la cirugía bariátrica $(\mathrm{CB})$ como un tratamiento efectivo para el descenso de peso en obesos severos; actualmente la evidencia disponible demuestra que la cirugía es la manera más efectiva para lograr la remisión de la diabetes tipo 2 (DM2) en pacientes obesos². En 2009, la American Diabetes Association (ADA) incluyó por primera vez a la CB como opción terapéutica en pacientes obesos con DM2 e inadecuado control metabólico con tratamiento médico convencional, y en el año 2011 la International Diabetes Federation (IDF), en su Position Statement estableció criterios de elección de CB en pacientes con DM2 e índice de masa corporal $>30 \mathrm{~kg} / \mathrm{m}^{2}$.

En algunos pacientes el fracaso de la terapéutica convencional hizo pensar en la cirugía bariátrica como un tratamiento capaz de revertir o mejorar la diabetes; nació de este modo el concepto de "cirugía metabólica" el cual hace referencia a "la cirugía que se realiza sobre el tubo digestivo con el objetivo de conseguir la mejoría o remisión de la DM2 y la reducción del riesgo cardiometabólico." Provee una alternativa terapéutica para la insulinorresistencia y la distribución del tejido adiposo presente en estos pacientes y predice un resultado favorable con disminución a largo plazo de la mortalidad cardiovascular secundaria a $\mathrm{DM}^{3}$, en la cual se produce un deterioro progresivo de la función de las células de los islotes pancreáticos, sobreviene una reducción en la síntesis de insulina, sumado al aumento de la resistencia a la insulina por parte de los tejidos periféricos que afectan y deterioran la homeostasis de la glucemia.

La rápida mejora del control glucémico observada luego de un procedimiento bariátrico se debería a cambios en la masa de células beta, deten- ción de la apoptosis, estimulación de la replicación celular y neogénesis en respuesta a diferentes estímulos como son la restricción calórica, el rápido pasaje de alimentos a nivel del íleon y la exclusión parcial del estómago y del intestino proximal (duodeno y parte del yeyuno).

La cirugía metabólica en pacientes que cumplen estrictamente los criterios de selección puede lograr la remisión parcial, completa y/o prolongada de la diabetes lo cual supone un avance significativo en su tratamiento, en tanto que la indicación está dirigida a pacientes con fracaso por más de un año con tratamiento médico según las guías vigentes. Se debe emplear el concepto de remisión y no el de curación en la evaluación de los resultados en la cirugía metabólica ${ }^{4}$.

La cirugía bariátrica -cada vez más frecuente en mujeres más jóvenes, en etapas reproductivasobliga a programar el embarazo y realizar controles previos y durante la gestación que eviten los riesgos de complicaciones materno-fetales. La tasa de infertilidad en mujeres obesas es mayor y mejora significativamente con el descenso de peso.

En el embarazo aumentan las demandas de macro y micronutrientes que aseguren un saludable crecimiento y desarrollo feto-placentario; en las pacientes sometidas a CB deberá tenerse en cuenta que la concepción debe evitarse en los 18 meses posteriores a la realización de la misma dado el riesgo nutricional durante ese período, ya que en el primer año de post-operatorio se considera que se encuentra en una fase catabólica en virtud de la gran pérdida de peso y, según algunos autores, aumenta el riesgo de fetos de bajo peso para la edad gestacional y/o partos prematuros ${ }^{5}$.

Se deberá tener en cuenta especialmente el aumento de peso durante la gesta y la adecuación de la pesquisa de diabetes gestacional en pacientes que, en virtud de la $\mathrm{CB}$, tienen alterado el ritmo de evacuación gástrica así como la absorción de macro y micronutrientes ${ }^{5}$.

Finalmente creemos que debe considerarse la necesidad de valorar la incorporación racional de 
la CB en el contexto de un equipo multidisciplinario con experiencia como un recurso terapéutico en los algoritmos de tratamiento escalonado de la
DM2, incluso tener en cuenta la programación del embarazo y el cuidado durante la gesta, el parto y el puerperio en estas pacientes.

Dra. Susana Gutt

\section{REFERENCIAS}

1. James WP. WHO recognition of the global obesity epidemic. International Journal of Obesity (2005) 2008, 32 Suppl. 7, S120-126.

2. Courcoulas A, Yanovski S, Bonds D, et al. Long-term outcomes of bariatric surgery. A National Institute of Health Symposium. JAMA Surg. Published online, october 1, 2014.

3. Cohen R. Metabolic surgery: who and when? Is there a good answer? Nutr. Hosp. 2013; 28 (Supl): 14-16.

4. Boza C, Valdreas P, Daroch D, et al. Metabolic surgery: Roux-en-Y gastric bypass and variables associated with diabetes remission in patients with $\mathrm{BMI}<35$. Obes. Surg. 2014; 24: 1391-1397.

5. Karmon A, Shelner E. Pregnancy after bariatric surgery: a comprehensive review Arch. Ginecol. Obstet. 2008; 277: 381-388.

Nota del Comité Editorial: los artículos editoriales reflejan exclusivamente la opinión de su autor. 